\title{
Percepção da enfermagem sobre a adesão e o abandono do tratamento da tuberculose
}

Perception of the Nursing team on the adherence and abandonment of tuberculosis treatment

Percepción de la enfermería sobre la adherencia y el abandono del tratamiento de la tuberculosis

\section{Ana Paula Vanzetto Simeão Freire ${ }^{\mathrm{I}}$, Karina Amadori Stroschein Normann" ${ }^{\mathrm{II}}$ Priscila Tadei Nakata $^{\text {III }}$, Dayane de Aguiar Cicolella ${ }^{\text {IV }}$}

\begin{abstract}
Resumo: Objetivo: conhecer a percepção da enfermagem sobre os fatores envolvidos na adesão e no abandono do tratamento da tuberculose. Método: estudo qualitativo, descritivo-exploratório realizado no Centro de Referência em Tuberculose no município de Porto Alegre no Rio Grande do Sul. Nove profissionais da enfermagem responderam à entrevista semiestruturada e foi realizada a análise de conteúdo de Bardin. Resultados: identificou-se quatro categorias temáticas: a organização do serviço e o papel da enfermagem no processo de trabalho; a raiz social da tuberculose; a consulta de enfermagem como artifício para o fortalecimento da adesão ao tratamento; rede de serviços como estratégia para reduzir o abandono do tratamento. Conclusão: os profissionais de enfermagem reconhecem sua responsabilidade na adesão ao tratamento da tuberculose e percebem no cotidiano de trabalho os fatores que influenciam o abandono. Reconhecem a necessidade de um trabalho em rede, intersetorial e de proteção social para o controle da doença.

Descritores: Tuberculose; Enfermagem; Tratamento farmacológico; Recusa do paciente ao tratamento; Adesão à medicação
\end{abstract}

\begin{abstract}
Objective: to know the perception of the nursing team about the factors involved in adherence and abandonment of tuberculosis treatment. Method: qualitative, descriptive-exploratory study carried out at the Tuberculosis Reference Center in the city of Porto Alegre, in Rio Grande do Sul. Nine nursing professionals responded to the semistructured interview and Bardin's content analysis was carried out. Results: four thematic categories were identified: the organization of the service and the role of the nursing team in the work process; the social root of tuberculosis; nursing consultation as a device for strengthening treatment adherence; service network as a strategy to reduce treatment abandonment. Conclusion: nursing professionals recognize their responsibility in the adherence to the treatment of tuberculosis and perceive the factors that influence abandonment in their daily work. They recognize the need for a networked, intersectoral, and of social protection effort to control the disease.
\end{abstract}

\footnotetext{
${ }^{\text {I }}$ Enfermeira, Especialista em Saúde Pública e PSF, Secretaria Municipal de Saúde, Porto Alegre, RS, Brasil, apaulavs@gmail.com, ORCID: 00000001-7095-4713

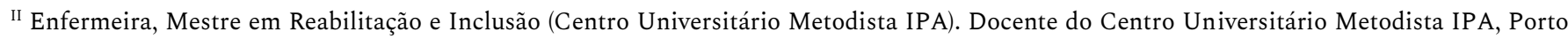
Alegre, RS, Brasil, karina.stroschein@gmail.com, ORCID: 0000-0003-4962-3279

III Enfermeira, Mestre em Enfermagem (PPGEnf-UFRGS), Secretaria Estadual de Saúde, Departamento de Coordenação dos Hospitais Estaduais, Hospital Sanatório Partenon, Porto Alegre, RS, Brasil, priscilanakata@gmail.com, ORCID: 0000-0001-6181-8381

IV Enfermeira, Doutoranda e Mestre em Enfermagem (PPGEnf-UfRGS), Centro Universitário Metodista IPA e CESUCA Faculdade, Porto Alegre, RS, Brasil, dayane.cicolella@gmail.com, ORCID: 0000-0001-7912-1216
} 
Percepção da enfermagem sobre a adesão e o abandono do tratamento da tuberculose | 2

Descriptors: Tuberculosis; Nursing; Pharmacological treatment; Patient's refusal of treatment; Adherence to medication

Resumen: Objetivo: conocer la percepción de la enfermería sobre los factores implicados en el cumplimiento y abandono del tratamiento antituberculoso. Método: estudio cualitativo, descriptivo-exploratorio realizado en el Centro de Referencia de Tuberculosis en la ciudad de Porto Alegre, Rio Grande do Sul. Nueve profesionales de enfermería respondieron a la entrevista semiestructurada y se realizó el análisis de contenido de Bardin. Resultados: se identificaron cuatro categorías temáticas: la organización del servicio y el papel de la enfermería en el proceso de trabajo; la raíz social de la tuberculosis; consulta de enfermería como dispositivo para fortalecer la adherencia al tratamiento; red de servicios como estrategia para reducir el abandono del tratamiento. Conclusión: los profesionales de enfermería reconocen su responsabilidad de adherirse al tratamiento de la tuberculosis y perciben los factores que influyen en el abandono en su trabajo diario. Reconocen la necesidad de redes, protección intersectorial y social para controlar la enfermedad.

Descriptores: Tuberculosis; Enfermería; Tratamiento farmacológico; Rechazo del tratamiento por parte del paciente; Adherencia a la medicación

\section{Introdução}

O recrudescimento da tuberculose $(\mathrm{TB})$, doença infecciosa de transmissão aérea causada pelo Complexo Mycobacterium Tuberculosis, continua sendo um grave problema de saúde pública. O grande desafio é quebrar a cadeia de transmissão da doença, cuja fonte são as pessoas com TB pulmonar bacilífera, que ao falarem, tossirem ou espirrarem eliminam os bacilos no ar ambiente. ${ }^{1}$ No ano de 2017, das aproximadamente 10 milhões de pessoas que foram acometidas pela doença no mundo, 1,3 milhões foram a óbito. ${ }^{2} \mathrm{E}$, apesar de $60 \%$ dos novos casos de TB mundiais estarem concentrados em países do continente asiático e africano, o Brasil concentra 33\% da carga de TB das Américas. ${ }^{1}$ Em 2018, o coeficiente de incidência, referente a média nacional de casos foi de $34,8 / 100.000$ habitantes e a mortalidade pela doença chegou a 2,2/100.000 habitantes em 2017. No mesmo ano, o Rio Grande do Sul (RS) ocupou o quarto lugar em incidência de casos, apresentando um coeficiente de 40/100.000 habitantes dentre as unidades federadas. ${ }^{2}$ Essa alta incidência da doença associada aos elevados índices de abandono do tratamento coloca o Brasil entre os países prioritários para o controle da TB. Tal cenário epidemiológico nacional insatisfatório é agravado pela concentração dos casos da doença nas populações vulneráveis: pessoas vivendo em situação de rua (PSR), pessoas privadas de liberdade (PPL), pessoas vivendo com HIV/Aids (PVHIV) e indígenas. ${ }^{1}$ 
Para dar conta deste cenário o Ministério da Saúde (MS), juntamente com as demais instâncias governamentais, tem direcionado as ações de enfrentamento da TB de acordo com a atual estratégia pelo fim da TB (End TB Strategy), que tem como objetivo o fim da epidemia global da doença. ${ }^{3}$ Nessa perspectiva, e em consonância com o Plano Nacional pelo Fim da TB como problema de saúde pública, o Programa Nacional de Controle da TB (PNCT) preconiza ações a partir de três pilares: prevenção e cuidado integrado centrado no paciente, políticas arrojadas e sistemas de apoio e intensificação da pesquisa e inovação. Esses pilares constituem a base para a urgência em desenvolver e qualificar estratégias de fortalecimento da adesão terapêutica. Dentre as quais, destacam-se o acolhimento, o Tratamento Diretamente Observado (TDO) e o Projeto Terapêutico Singular (PTS). ${ }^{1}$

Em relação à organização e implementação das principais estratégias de fortalecimento da adesão, as atuais recomendações para o controle da TB destacam o protagonismo das ações de enfermagem. Essa categoria profissional segue sendo atuante tanto em território nacional, quanto em países pobres com alta carga de TB drogarresistente. ${ }^{4-5}$ Atualmente, o desafio que surge a estes profissionais extrapola aspectos da assistência, gerenciamento e organização dos serviços. Nesse sentido, as estratégias de fortalecimento da adesão ao tratamento exigem ações de saúde por meio da articulação intersetorial e o enfrentamento das situações de vulnerabilidade que acometem a maior parte das pessoas com TB. ${ }^{6}$

Frente ao exposto, este estudo busca conhecer a percepção da enfermagem sobre os fatores envolvidos na adesão e no abandono do tratamento da tuberculose.

\section{Método}

Trata-se de um estudo descritivo e exploratório com abordagem qualitativa, realizado em um serviço de referência secundária e terciária para o tratamento da TB no município de Porto Alegre, 
Percepção da enfermagem sobre a adesão e o abandono do tratamento da tuberculose | 4

Rio Grande do Sul, Brasil. Os participantes foram profissionais da enfermagem envolvidos no atendimento e tratamento dos usuários com TB. Os critérios de inclusão foram a atuação do profissional de enfermagem no serviço durante a coleta de dados e apresentar experiência por mais de seis meses no tratamento da doença. Os critérios de exclusão foram não fazer parte do quadro de funcionários permanentes do serviço ou estar afastado de suas funções por motivo de férias ou licença no período da coleta de dados. Dessa forma, após a aplicação dos critérios expostos, do total de 12 profissionais de enfermagem permaneceram incluídos nessa pesquisa nove participantes.

A coleta de dados ocorreu em maio de 2018. A pesquisadora entrou em contato com a coordenação do serviço para apresentar a pesquisa e agendar um encontro presencial com cada um dos profissionais de enfermagem. Neste encontro, era realizado o convite para a participação da pesquisa, bem como assinado o Termo de Consentimento Livre e Esclarecido, àqueles que aceitavam participar. Após, em sala reservada de forma a garantir a privacidade dos participantes, a pesquisadora realizou a entrevista. Primeiramente, foram coletadas informações de caracterização dos participantes como registro da categoria profissional, sexo, idade e tempo de atuação no atendimento da TB. Para avaliar a percepção dos profissionais de enfermagem quanto aos fatores relacionados a adesão e ao abandono do tratamento foi utilizado um roteiro de entrevista semiestruturada, com as seguintes perguntas abertas: De que forma a enfermagem está envolvida no processo de orientação dos usuários acerca do tratamento da TB? Na sua opinião, quais os motivos que levam os usuários ao abandono do tratamento da TB? As estratégias utilizadas para evitar o abandono do tratamento da TB necessitam de mudanças? Em caso afirmativo, quais seriam essas mudanças? Como o trabalho da enfermagem poderia colaborar para estimular os usuários ao não abandono do tratamento da TB? Todas as entrevistas foram gravadas na íntegra.

Em relação à análise do material, foi aplicado o método de Análise de Conteúdo de Bardin. ${ }^{7}$ Para tal, foi realizada a organização da análise, a codificação, a categorização e o tratamento dos resultados por meio de inferências. Após a coleta de dados, a pesquisadora realizou a transcrição de 
5 | Freire APVS, Normann KAS, Nakata PT, Cicolella DA

todas as entrevistas na íntegra e a codificação deste material. Na sequência, foi possível a realização de inferências acerca dos achados. Para a diferenciação e preservação da identidade dos participantes foram utilizados códigos com as seguintes letras (seguidos pelo número de ordem da realização das entrevistas): E para os profissionais enfermeiros, TE para os técnicos de enfermagem e AE para o auxiliar de enfermagem. Por exemplo: TE1, TE2, E3, E4, AE5, TE6, TE7, TE8 e E9.

O projeto da presente pesquisa foi aprovado pelo Comitê de Ética da Escola de Saúde Pública/CES/RS, em 09 de maio de 2018, sob o parecer nº 2.646.027, CAAE 79992817.4.3002.5312, pelo Comitê de Ética e Pesquisa do Centro Universitário Metodista IPA, sob o parecer no 2.560.294, CAAE 79992817.4.0000.5308 e pela Comissão Técnica e Científica do local onde foi realizada a pesquisa. Este estudo atendeu as exigências técnicas e científicas que regulamentam os preceitos éticos determinados pela Resolução $N^{\circ}$ 466/2012, do Conselho Nacional de Saúde que dispõe sobre a ética de pesquisa envolvendo seres humanos.

\section{Resultados e discussão}

Dos nove profissionais da enfermagem entrevistados, três eram enfermeiras, cinco técnicos de enfermagem e um auxiliar de enfermagem. Oito do sexo feminino e um do sexo masculino. A faixa etária variou de 34 a 65 anos. A média de tempo no exercício profissional no atendimento da TB foi de 14 anos.

Em relação à análise, foi possível identificar quatro categorias temáticas: a organização do serviço e o papel da enfermagem no processo de trabalho; a raiz social da tuberculose; a consulta de enfermagem como artifício para o fortalecimento da adesão ao tratamento; rede de serviços de saúde como estratégia para reduzir o abandono ao tratamento.

\section{Organização do serviço e o papel da enfermagem no processo de trabalho}


Percepção da enfermagem sobre a adesão e o abandono do tratamento da tuberculose | 6

Os profissionais percebem que exercem atividades nas diferentes etapas do atendimento da TB, sentem-se corresponsáveis pelo sucesso do tratamento. Identificam que exercem um cuidado que vai desde a recepção dos usuários e o fornecimento de orientações até a conclusão do tratamento.

A enfermagem está à frente, dá toda a orientação [...] mas a gente procura sempre incentivar eles [usuários]. (TE2)

A enfermagem deve abordar as questões da doença em si e a forma de transmissão, tratamento, tempo de duração, os medicamentos que são utilizados pelo paciente e seus paraefeitos. (E4)

Eu tento me envolver o máximo possível para fazer com que o paciente entenda o tratamento. (E3)

A atuação dos profissionais de enfermagem em várias etapas do atendimento da TB reforça o protagonismo desta categoria no enfrentamento da doença. A eles cabem ações de vigilância de base territorial como a busca de casos suspeitos da doença na comunidade para detecção precoce da doença. Ademais são responsáveis pela realização da assistência direta às pessoas em tratamento por meio do fornecimento de orientações sobre a doença para esclarecimento de dúvidas do usuário e familiares. Ainda, a identificação de possíveis contatos do indivíduo com TB e a supervisão da tomada de medicação por meio do TDO, fazem parte dos cuidados prestados. ${ }^{8}$ Além disso, é importante ressaltar que, o envolvimento e protagonismo do indivíduo na condução do seu tratamento, claramente percebido pelos profissionais, vem ao encontro do preconizado pelo atual plano nacional de controle da TB. ${ }^{3}$

Sobre o envolvimento da equipe de enfermagem na adesão terapêutica, os profissionais entrevistados percebem o acolhimento como um dos principais elementos para o sucesso dos tratamentos. Compreendem que acolher reflete de forma positiva na relação terapêutica.

O fundamental é o acolhimento do paciente [...] nós tínhamos uma paciente que era muito rebelde e ela deixou de faltar à consulta, porque cada vez que ela faltava a médica pegava o telefone e ligava [...] e ela achou o máximo aquilo. Se 
sentiu importante [...] no momento que tu demonstras interesse pelo tratamento deles, é importante. (TE6)

Em um primeiro momento a gente acolhe. (TE7)

A percepção do acolhimento como primordial na adesão vai ao encontro do preconizado pelo primeiro pilar, que sustenta a atual estratégia pelo fim da $\mathrm{TB}$, a prevenção e o cuidado integrado e centrado no paciente. ${ }^{3} \mathrm{~A}$ intenção é a realização de um cuidado integrado e centrado no paciente, por meio de um bom relacionamento interpessoal, para acionar vínculos terapêuticos singulares ${ }^{9-10}$ potencialmente capazes de melhorar a adesão ao tratamento da TB. No atual cenário de enfrentamento da TB, o MS preconiza o tratamento das populações vulneráveis como prioritário. Nesse sentido, o acolhimento se torna uma importante tecnologia a ser utilizada pelos profissionais de enfermagem para o fortalecimento da adesão. ${ }^{1}$ Uma escuta ativa e acolhedora facilita a compreensão dos principais componentes subjetivos que influenciam no comportamento das pessoas com TB. Dentre eles, os diferentes significados que a pessoa sustenta sobre doença, o grau de envolvimento e as expectativas em relação ao tratamento prescrito são importantes para o planejamento de um cuidado compartilhado pelo enfermeiro. ${ }^{11-12}$ Os profissionais de enfermagem também compreendem que o usuário é corresponsável pelo seu estado de saúde.

Eu tento me envolver o máximo possível para fazer com que o paciente entenda o tratamento. (E3)

Eu costumo brincar com os pacientes que o tratamento é $50 \%$ nós, a instituição, a medicação, o atendimento, os exames e 50\% é o dele com o tratamento. [...] se ele assumir o papel de protagonista do tratamento dele, nós temos uma chance maior de atingir o sucesso. (TE6)

A percepção dos profissionais de enfermagem quanto ao entendimento de que parte da responsabilidade pelo tratamento é do indivíduo com TB está em conformidade com a atual definição de adesão utilizada pelo MS. Ela se configura como um processo colaborativo em que a pessoa em tratamento deve aceitar a instituição de determinado regime terapêutico. ${ }^{3}$ Esta postura colaborativa 
Percepção da enfermagem sobre a adesão e o abandono do tratamento da tuberculose $\mid 8$

pode ser observada nos casos em que o tratamento se dá pelo TDO, modalidade em que o usuário se responsabiliza e se compromete em comparecer à Unidade de Saúde cinco ou três vezes na semana para tomada da medicação de forma supervisionada pelos profissionais de saúde. ${ }^{8}$ No entanto, sem desconsiderar, que ao indivíduo cabe parte da responsabilidade pelo tratamento.

Alerta-se que, em se tratando das populações vulneráveis, é necessário ter cautela na exaltação da responsabilidade individual frente ao tratamento de uma doença. Isso porque essas populações, muitas vezes, estão desprovidas de condições básicas de vida decorrentes de violação de direitos humanos que as impedem de “colaborar por si só” com o tratamento. Fato que, requer maior atenção das ações de proteção social realizadas pelo governo, pois os programas de transferência de renda, como o Bolsa Família, apresentaram efeito positivo no tratamento da doença. ${ }^{13}$

Ações de educação também são percebidas pela equipe como fundamentais para o sucesso do tratamento.

Eu acho que a primeira questão é a informação. A tuberculose tem que fazer sentido para eles. O tratamento tem que fazer sentido. O que é essa doença? Por que eu devo tratar? Por que eu não devo abandonar? E acho que é muito importante da enfermagem trabalhar essas questões educacionais com o paciente. Em a doença fazendo sentido inicialmente, já é um grande avanço no tratamento, porque ele vai entender os motivos pelos quais ele não pode abandonar o tratamento. (E4)

Embora as ações de educação em saúde sejam reconhecidas pela equipe de enfermagem no fortalecimento da adesão ao tratamento da TB, pesquisa reforça que, por vezes, estas ainda se mostram pontuais e pouco expressivas no processo de trabalho das equipes da Atenção Básica. ${ }^{14}$ Isso porque abordagens que desconsideram o diálogo tendem a delegar aos indivíduos toda a responsabilidade pelo seu estado de saúde. O que por sua vez, contribui para a culpabilização pela não adesão ao tratamento. Sendo assim, entende-se que ações de educação em saúde, no contexto da TB, possam ser mais efetivas quanto mais considerarem a interação entre o saber técnico dos profissionais com o saber popular das pessoas em tratamento. 


\section{A raiz social da Tuberculose}

Dentre os fatores relacionados ao abandono do tratamento da $T B$, as condições socioeconômicas dos usuários foram as mais referidas pelos profissionais de enfermagem.

É uma doença de profunda raiz social [...] A questão do estigma da tuberculose está muito ligada ao abandono do tratamento. (E4)

[...] são vários fatores. Um deles é a condição social deles. Ele não tem onde morar, não tem o que comer, não tem apoio da família. Se sentem constrangidos com a doença. Ainda existe preconceito da doença. (AE5)

Existe o abandono por questões que a gente pode atribuir às condiçôes socioeconômicas, uso de drogas e álcool, questôes de vulnerabilidade tanto individual quanto social. (E9)

Nós temos pacientes que abandonaram pelo simples fato de que não tinham como vir porque trabalhavam e os horários não fechavam para fazer os injetáveis [...] ele disse: eu tenho que trabalhar ou vou ser despejado. (TE6)

Apesar de ser amplamente conhecida a associação entre a ocorrência de TB com más condições socioeconômicas, é a primeira vez que a necessidade de enfrentamento dos determinantes sociais da saúde é formalizada como recomendação nos manuais de controle da doença. ${ }^{3}$ Tal fato decorre, em parte, das crescentes desigualdades sociais do Brasil, um dos países com maior concentração de renda e iniquidades em saúde, condições que agravam as taxas de abandono do tratamento. Tais aspectos se mostram ainda mais preocupantes na PPL, as quais convivem em celas superpopulosas e desprovidas de atendimento de saúde. ${ }^{15-18}$ Apesar do entendimento da necessidade de enfrentamento desses determinantes sociais, é preciso atentar-se para não superestimar a efetividade do setor saúde na resolução de “problemas macroestruturais”. Isso porque as ações dos profissionais de enfermagem serão mais efetivas quanto mais se somarem às ações de outros setores. Nesse contexto, é preciso valorizar o trabalho da enfermagem no envolvimento da família como rede 
de apoio ao usuário em tratamento. Uma rede de apoio familiar auxilia na redução do estigma, da discriminação e do isolamento social gerado pela doença. ${ }^{19}$

As questões subjetivas da pessoa em relação ao tratamento também foram sinalizadas pelos profissionais entrevistados como fatores que dificultam o processo de adesão.

Mas também tem questões subjetivas do paciente. Quando eu atendo o paciente que abandona e que retorna eu escuto muito assim: Ah! Eu parei de tomar o remédio porque eu não me sinto doente [...] mas tem também o outro lado que eu escuto muito: eu estou bem, não preciso mais tomar o remédio [...] o paciente não se identifica doente. (E9)

Tal relato corrobora a importância em se considerar os aspectos subjetivos do usuário no tratamento da TB, pois a forma singular como cada indivíduo compreende o processo saúde-doença influencia na adesão ao tratamento. ${ }^{19}$ Em relação a isso, estudo que avaliou os fatores subjetivos relacionados à adesão terapêutica em usuários de um centro de referência, reforça que a importância da realização de mais pesquisas com abordagem qualitativa. ${ }^{5}$

\section{A consulta de enfermagem como artifício para o fortalecimento da adesão ao tratamento}

Nos depoimentos dos profissionais, percebe-se que o fortalecimento da adesão terapêutica demanda tempo da equipe e, principalmente, do enfermeiro durante a consulta de enfermagem. Entretanto, a realidade dos serviços e a falta de recursos humanos é uma realidade no cotidiano de trabalho dos serviços de saúde que lidam com tal agravo.

$\grave{A}$ s vezes eu tenho vontade de fazer mais, mas eu sei que as estruturas não me ajudam. A gente esbarra no sistema. (E3)

Aqui a gente faz a consulta de enfermagem no início do tratamento e quando ele retorna para resgatar um tratamento abandonado. $\dot{A}$ s vezes eu acho que a gente deveria fazer uma consulta de enfermagem de adesão, toda a vez que o paciente vem [...] porque quando chega lá no $12^{\circ}$ mês, ainda tem mais 6 meses ou 1 ano de tratamento [nos casos de drogarresistência], mas ele está super bem. Teria de ter consultas com outras demandas, no sentido de reforçar: o senhor está bem, mas não está curado. Claro que a gente não tem pernas para isso. (E9) 
A consulta de enfermagem, atividade privativa do enfermeiro, traz consigo a potencialidade de fortalecimento da adesão ao tratamento da TB. Tal atividade se configura como um espaço de ampliação das abordagens estritamente biomédicas, pois inclui a pluralidade do cuidado proposto pela saúde coletiva. ${ }^{20}$ No entanto, apesar de sua importância, a consulta de enfermagem é mais uma das inúmeras atividades do enfermeiro, que, muitas vezes, precisa dividir o seu tempo de trabalho no planejamento e gerenciamento das ações de saúde no serviço.

Percebe-se, também, certa dificuldade dos profissionais de enfermagem em lidar com os usuários que parecem não compreender a real dimensão da necessidade de seguir o tratamento da TB. Nesse aspecto, a consulta de enfermagem é percebida como um espaço de acolhimento que deve ser incorporado periodicamente no seguimento do tratamento.

E outra coisa que eu também acho é a falta de conhecimento da doença. Não tem muito interesse pela sua saúde [...]. (E3)

Eu escuto muito assim: Ah! Eu parei de tomar o remédio porque eu não me sinto doente [...] eu estou bem, não preciso mais tomar o remédio. (E9)

Por ser um paciente que faz um tratamento muito longo, não adianta fazer só no início do tratamento [a consulta de enfermagem]. É um trabalho que tem de ser em todas as consultas [médicas]. Vai se tornar um pouco repetitivo? Vai, mas é importante insistir no acolhimento, na importância do comprometimento dele com o tratamento, justamente por ser muito longo. (TE6)

Apesar da importância da consulta de enfermagem enquanto espaço, tecnologia de cuidado e ferramenta capaz de qualificar o manejo da TB, é preciso considerar algumas limitações. ${ }^{21-22}$ Tais limitações se referem a fragmentação existentes entre as etapas de anamnese, exame físico, diagnósticos, planejamento, implementação e avaliação do plano terapêutico. Quanto a esses aspectos, estudo que avaliou a consulta de enfermagem aos pacientes com TB na Atenção Básica, demonstrou que a anamnese e o exame físico eram realizados, mas que nem sempre as outras etapas eram incluídas nas consultas. ${ }^{23}$ Entende-se que cabe ao próprio profissional enfermeiro, bem como 
aos gestores dos serviços de saúde, a valorização dessa atividade privativa, de forma a integrá-la de modo contumaz às atividades de controle da TB.

\section{Rede de serviços como estratégia para reduzir o abandono do tratamento}

Para os profissionais de enfermagem entrevistados, o sucesso do tratamento está fortemente relacionado ao acesso à rede de serviços de saúde e, consequentemente, ao envolvimento das diversas categorias profissionais.

Precisa de mais pessoas na busca desses pacientes [faltosos] quando começam o tratamento, porque eles não vêm. E ficam 3, 4 meses [longe do tratamento]. (TE1)

Acredito que a gente deveria ter um reforço social junto com a clínica da tuberculose, porque a gente tem visto que a saúde, por si só, não tem conseguido dar conta dessa doença. (E4)

Acredito que muita coisa é feita só dentro da saúde. Então, a gente que é da saúde, atende, tem estratégias de busca, tem estratégias de evitar o abandono, mas não consegue extrapolar o campo da saúde. Não tem uma rede que possa ter um encaminhamento direto a um serviço de acompanhamento, um serviço social da região [...] eu acho que a gente é muito limitado enquanto saúde. (E9)

A realização de ações intersetoriais emerge como importante recomendação para o enfrentamento dos determinantes sociais relacionados à TB. ${ }^{12} \mathrm{O}$ atual manual de controle da doença destaca que a aproximação entre o setor saúde e a assistência social auxilia na redução das situações de vulnerabilidades de grande parte das pessoas em tratamento. ${ }^{3}$ No entanto, apesar do reconhecimento da importância da intersetorialidade, é preciso problematizar o quanto as suas ações deveriam ou conseguiriam ser efetivas na resolução de problemas sociais gerados pelas próprias desigualdades dos sistemas econômicos vigentes. ${ }^{16}$ Nesse sentido, a acredita-se que, delegar à intersetorialidade a potencialidade de reduzir situações de pobreza é superestimar as suas contribuições para além da qualificação do cuidado em saúde. Isso por que a redução das desigualdades necessita de mudanças macroestruturais que vão além das ações de diferentes setores. ${ }^{24}$ 
Além disso, também é percebido que, embora os serviços de referência possuam uma equipe interdisciplinar na assistência direta aos usuários, boa parte destes indivíduos com TB tem dificuldade de acesso a profissionais da psicologia, psiquiatra e serviço social.

Eu acho que tinha de haver uma aproximação maior entre saúde e a rede. Acho que uma das coisas que dificulta isso é a questão da espera. Nós [serviço de referência] sermos estado e eles [atenção básica] município, acho que dificulta. Mas é como se a gente [serviço de referência] ficasse separado de tudo. Então, nós fazemos as nossas buscas, temos serviço social que faz o contato, que faz a visita, mas não conseguimos conversar, por exemplo, com o profissional lá da atenção básica que faz a visita do bolsa familia, que poderia ajudar na adesão ao tratamento, com o agente comunitário de saúde. (E9)

A complexidade que envolve a adesão ao tratamento da TB torna urgente a participação de várias categorias profissionais, reforçando a importância da organização de uma rede de serviços sólida e resolutiva para um desfecho positivo dos casos. ${ }^{21}$ Quanto a esse aspecto, no atual contexto político, é preciso atentar-se não somente para a necessidade de qualificação dos serviços do Sistema único de Saúde (SUS), mas também pela sua própria defesa e manutenção enquanto serviço público de saúde de acesso a todos. Isso porque a sustentabilidade desses serviços depende tanto da formação dos profissionais que neles atuam, quanto da formulação de políticas que contemplem a inclusão e participação das várias profissões. ${ }^{25}$

Ainda sobre a rede de serviços no atendimento da $\mathrm{TB}$, conforme relatos, os profissionais identificam o desejo de integração entre as etapas da busca do sintomático respiratório (pessoa que tosse por tempo igual ou superior a 3 semanas), a realização do diagnóstico e o acompanhamento do tratamento. Tal integração se refere tanto às ações dentro de um único serviço de saúde quanto na referência e contrarreferência nos diferentes níveis de atenção à saúde (Atenção Básica e Serviço Especializado).

A busca na atenção básica e uma parceria melhor da referência com a atenção básica. Acho que as equipes tinham de ser praticamente uma coisa só. Trabalhar em ação conjunta. (E3) 
Percepção da enfermagem sobre a adesão e o abandono do tratamento da tuberculose | 14

A gente faz alguns encaminhamentos, mas é como se as vezes eu sentisse que a gente não faz parte da rede como um todo. Então, a gente encaminha para o posto de saúde e o posto de saúde, a partir de então, tem que dar o encaminhamento para a psicologia, para o serviço social, e enfrenta a dificuldade de falta desses serviços lá também. (E9)

No Brasil, apesar da construção da Linha de Cuidado para Tuberculose que inclui a descentralização do tratamento para a Atenção Básica, ${ }^{1}$ ainda há dificuldade de comunicação entre os diferentes níveis da assistência. Para tal, existe um esforço no sentindo de capacitar os profissionais que atuam na ponta para o manejo da doença. ${ }^{20}$ Tal capacitação deve levar em consideração que, por se tratar de um agravo fortemente associado às situações de vulnerabilidade, é necessário a realização de um cuidado integrado e não fragmentado. ${ }^{17}$ Além disso, destaca-se que a falta de apoio e comprometimento dos gestores na alocação de recursos financeiros e humanos, pode vir a comprometer a capacidade da Atenção Básica em manejar casos de TB. O que por sua vez refletirá em índices epidemiológicos aquém dos preconizados pelo MS.

\section{Considerações finais}

Os profissionais de enfermagem envolvidos no processo do cuidado dos indivíduos com TB reconhecem a responsabilidade e a importância do trabalho de orientação e acompanhamento do tratamento dos usuários. Também percebem, nesse contexto, diversos fatores que podem influenciar a adesão e o abandono do tratamento da doença. Nesse aspecto, o acolhimento destacou-se como importante fator para a construção de um vínculo com o usuário, que poderá se refletir positivamente não só na relação terapêutica, mas principalmente no controle da TB. Salienta-se que a equipe de enfermagem observa o abandono do tratamento como um reflexo das questões relacionadas aos determinantes sociais da saúde e consideram este um grande desafio a ser enfrentado. Nesse sentido, a inexistência ou a insuficiência de medidas de proteção social para os usuários tendem a 
15 | Freire APVS, Normann KAS, Nakata PT, Cicolella DA

comprometer a qualidade do tratamento de uma doença com forte associação aos contextos de pobreza.

Pode-se observar, também, que apesar da percepção da necessidade da consulta de enfermagem de forma periódica durante todo o tratamento, existe uma limitação colocada pela sobrecarga de trabalho desta categoria profissional. Além disso, a realização de um cuidado em rede esbarra em dificuldades como a fragilidade da relação entre saúde e assistência social. Aspecto que, se somado a falta de uma rede familiar de apoio, pode vir a contribuir para o abandono do tratamento, dificultando ainda mais o controle da TB.

Sendo assim, os profissionais de enfermagem percebem-se com atribuições para além de questões biomédicas, que extrapolam o setor saúde, como a redução das situações de vulnerabilidade social (condições socioeconômicas desfavoráveis, estar em situação de rua, ser usuário de álcool e outras drogas) nas quais grande parte dos usuários com TB se encontra. Portanto, acredita-se que o equilíbrio entre a realização das ações desses diferentes setores e atores seja determinante para o controle da doença nos diferentes cenários e contextos sociais.

Como fator limitador desta pesquisa, considera-se o fato da mesma ter ocorrido com um “n” reduzido, o que impede que os dados sejam generalizados. No entanto, os resultados apresentam informações relevantes que poderão orientar novos estudos com o objetivo de ampliar a abrangência para outros níveis de atenção, com o intuito de corroborar os resultados encontrados.

\section{Referências}

1. Ministério da Saúde (BR), Secretaria de Vigilância em Saúde, Departamento de Vigilância Epidemiológica. Manual de recomendações para o controle da tuberculose no Brasil [Internet]. Brasília (DF): Ministério da Saúde; 2016 [acesso em 2020 mar 16]. 364 p. Disponível em: http://portalarquivos2.saude.gov.br/images/pdf/2016/outubro/15/panorama_tuberculose_brasil_mortalidade.pdf

2. Ministério da Saúde (BR), Secretaria de Vigilância em Saúde. Brasil Livre da Tuberculose: evolução dos cenários epidemiológicos e operacionais da doença. Boletim Epidemiológico [Internet]. Brasília (DF): 
Percepção da enfermagem sobre a adesão e o abandono do tratamento da tuberculose | 16

Ministério da Saúde; 2019 [acesso em 2020 mar 16];50(9):1-18. Disponível em: https://portalarquivos2.saude.gov.br/images/pdf/2019/marco/22/2019-009.pdf

3. Ministério da Saúde (BR), Secretaria de Vigilância em Saúde, Departamento de Vigilância das Doenças Transmissíveis. Brasil Livre da Tuberculose: Plano Nacional pelo Fim da Tuberculose como Problema de Saúde Pública [Internet]. Brasília (DF): Ministério da Saúde; 2017 [acesso em 2020 mar 16]. 52p. Disponível em: http://bvsms.saude.gov.br/bvs/publicacoes/brasil_livre_tuberculose_plano_nacional.pdf

4. Stival JF, Carol LM, Cardoso, AM. Emergência da tuberculose multirresistente e extensivamente resistente: uma abordagem sobre o panorama atual. RESAP [Internet]. 2016 [acesso em 2018 abr 21];2(3):123-37. Disponível em: http://www.revista.esap.go.gov.br/index.php/resap/article/view/36

5. Beraldo AA, Andrade RLP, Orfão NH, Silva-Sobrinho RA, Pinto ESG, Wysocki AD, et al. Adherence to tuberculosis treatment in Primary Health Care: perception of patients and professionals in a large municipality. Esc Anna Nery Rev Enferm. 2017; 21( 4 ):e20170075. doi: http://dx.doi.org/10.1590/2177-9465EAN-2017-0075

6. Dixon J, Tameris M. A disease beyond reach: nurse perspectives on the past and present of tuberculosis control in South Africa. Anthropol South Africa. 2018;41(4):257-69. doi: https://doi.org/10.1080/23323256.2018.1526096

7. Bardin L. Análise de conteúdo. São Paulo (SP): Edições 70; 2011. 279 p.

8. Brunello MEF, Simiele-Beck MF, Orfão NH, Wysocki AD, Magnabosco GT, Andrade RLDP, et al. Atuação da enfermagem na atenção a uma condição crônica (tuberculose): análise de fontes secundárias. Rev Gaúch Enferm. 2015;36(N Esp):62-9. doi: https://doi.org/10.1590/1983-1447.2015.esp.56363

9. Corrêa VAF, Acioli S, Mello AS, Dias JR, Pereira RDM. Projeto Terapêutico Singular: reflexões para a enfermagem em saúde coletiva. Rev Enferm UERJ. 2016;24(6):e26309. doi: https://doi.org/10.12957/reuerj.2016.26309

10. Ministério da Saúde (BR), Secretaria de Atenção à Saúde. HumanizaSUS: política nacional de humanização: Brasília (DF): Ministério da Saúde; 2013.

11. Rêgo LP, Cunha FF, Rodrigues ILA, Nogueira LMV, Andrade NCO. Assistência humanizada de enfermagem às pessoas doentes com tuberculose: revisão integrativa 2002-2012. Rev Baiana Saúde Pública. 2014;38(3):738-59. doi: https://doi.org/10.22278/2318-2660.2014.v38.n3.a744

12. Serrano Gallardo MP. Intersectorality, key to address social health inequalities. Rev Latinoam Enferm. 2019;e3124. doi: http://dx.doi.org/10.1590/1518-8345.0000-3124

13. Oliosi JGN, Reis-Santos B, Locatelli RL, Sales CMM, Silva Filho WG, Silva KC, et al. Effect of the Bolsa Familia Programme on the outcome of tuberculosis treatment: a prospective cohort study. Lancet Respir Med. 2018;7(2):E219-26. doi: https://doi.org/10.1016/S2214-109X(18)30478-9 
14. Sá LD, Gomes ALC, Carmo JB, Souza KMJ, Palha PF, Alves RS. Educação em saúde no controle da tuberculose: perspectiva de profissionais da estratégia Saúde da Família. Rev Eletrônica Enferm. 2013;15(1):103-11. doi: https://doi.org/10.5216/ree.v15i1.15246

15. Valença MS, Possuelo LG, Cezar-Vaz MR, Silva PEA. Tuberculosis in Brazilian prisons: an integrative review of the literature. Ciênc Saúde Colet. 2016;21(7):2147-60. doi: http://dx.doi.org/10.1590/141381232015217.16172015

16. Souza PHG, Medeiros M. Working paper number 163: the concentration of income at the top in Brazil, 2006-2014 [Internet]. Brasília (DF): International Policy Centre for Inclusive Growth; 2017 [cited 2019 Oct 23]. Available from: http://ipcig.org/pub/eng/WP163_The_concentration_of_income_at_the_top_in_Brazil.pdf

17. Chirinos NEC, Meirelles BHS, Bousfield ABS. Relationship between the social representations of health professionals and people with tuberculosis and treatment abandonment. Texto \& Contexto Enferm. 2017;26(1):e5650015. doi: https://doi.org/10.1590/0104-07072017005650015

18. Maffacciolli R, Hahn GV, Rossetto M, Almeida CPB, Manica ST, Paiva TP, et al. Using the notion of vulnerability in the production of knowledge about tuberculosis: integrative review. Rev Gaúch Enferm. 2015;36(N Esp):247-53. doi: https://doi.org/10.1590/1983-1447.2015.esp.51537

19. Dantas CN, Santos VEP, Tourinho FSV. A consulta de enfermagem como tecnologia do cuidado à luz dos pensamentos de Bacon e Galimberti. Texto \& Contexto Enferm. 2016;25(1):e2800014. doi: https://doi.org/10.1590/0104-0707201500002800014

20. Maranha NB, Silva MCA, Brito IC. A consulta de enfermagem no cenário da atenção básica e a percepção dos usuários: revisão integrativa. Academus [Internet]. 2017 [acesso em 2018 jun 02];2(1). Disponível em: https://smsrio.org/revista/index.php/reva/article/view/246

21. Oliveira DRC, Enders BC, Vieira CENK, Mariz LS. Avaliação da consulta de enfermagem aos pacientes com tuberculose na atenção primária à saúde. Rev Eletrônica Enferm. 2016;18:e1153. doi: http://dx.doi.org/10.5216/ree.v18.32593

22. Salzani MGB, Oliveira SAC, Rocha MAZP, Jesus GJ, Gazetta CE, Vendramini SHF, et al. Diagnóstico de tuberculose: perspectiva do profissional de enfermagem da atenção básica. Rev Fam Ciclos Vida Saúde Contexto Soc. 2017;5(2):180-90. doi: https://doi.org/10.18554/refacs.v5i2.1791

23. Souza J, Oliveira KS, Ávila TT, Quadros SR, Zilly A, Silva-Sobrinho RA. Incidência da tuberculose e a correlação entre a realização do tratamento e a cura. Rev Enferm UFSM. 2018;8(4):637-48. doi: http://dx.doi.org/10.5902/2179769230534

24. Meyer DE, Alvarenga LFC, Andrade SS, Félix J, Damico JGS. Quem aprende o que e com quem? Educação permanente em saúde e intersetorialidade como desafios políticos-pedagógicos. Saberes Plurais Educ Saúde [Internet]. 2018 [acesso em 2020 mar 21];2(3):104-19. Disponível em: https://seer.ufrgs.br/saberesplurais/article/view/86577 
Percepção da enfermagem sobre a adesão e o abandono do tratamento da tuberculose $\mid 18$

25. Campos GWS. SUS: o que e como fazer? Ciênc Saúde Colet. 2018;23(6):1707-14. doi: https://doi.org/10.1590/1413-81232018236.05582018

\section{Autor correspondente}

Ana Paula Vanzetto Simeão Freire

E-mail: apaulavs@gmail.com

Endereço: Rua Campos Elíseos, 340/403 Porto Alegre-RS

CEP: $90870-020$

\section{Contribuições de Autoria}

\section{1 - Ana Paula Vanzetto Simeão Freire}

Concepção e planejamento do projeto de pesquisa, coleta, análise e interpretação dos dados, redação inicial. Contribuições para redação final, atualização bibliográfica, revisão gramatical e revisão crítica do artigo.

\section{2 - Karina Amadori Stroschein Normann}

Orientação da fase de concepção e planejamento do projeto de pesquisa e redação inicial. Revisão gramatical e revisão crítica do artigo.

\section{3 - Priscila Tadei Nakata Zago}

Contribuições para atualização bibliográfica, redação final, revisão gramatical e revisão crítica do artigo.

\section{4 - Dayane de Aguiar Cicolella}

Contribuições para redação final, revisão gramatical e revisão crítica do artigo.

\section{Como citar este artigo}

Freire APVS, Normann KAS, Nakata PT, Cicolella DA. Percepção da enfermagem sobre a adesão e o abandono do tratamento da tuberculose. Rev. Enferm. UFSM. 2020 [Acesso em: Anos Mês Dia]; vol.10 e37: 1-18. DOI:https://doi.org/10.5902/2179769239456 